\title{
Absolute cross sections for dissociative electron attachment to HCN and DCN
}

\author{
O. May, D. Kubala, and M. Allan \\ Department of Chemistry, University of Fribourg, Chemin du Musée 9, CH-1700 Fribourg, Switzerland
}

\begin{abstract}
Absolute partial cross sections for the formation of $\mathrm{CN}^{-}$in dissociative electron attachment to $\mathrm{HCN}$ and DCN have been measured using a time-of-flight ion spectrometer combined with a trochoidal electron monochromator to be $940 \mathrm{pm}^{2}$ for $\mathrm{CN}^{-} / \mathrm{HCN}$ and $340 \mathrm{pm}^{2}$ for $\mathrm{CN}^{-} / \mathrm{DCN}$ at peaks of the bands due to the ${ }^{2} \Pi$-shape resonance. The dissociative electron attachment bands were then recorded under higher resolution, $60 \mathrm{meV}$, with a trochoidal monochromator plus quadrupole mass filter combination and found to have a nearly vertical onset at the threshold energy and to peak at $1.85 \mathrm{eV}$. Broad structure was observed on the bands, assigned to formation of vibrationally excited $\mathrm{CN}^{-}$, from which the branching ratios could be determined to be $1,0.49$, and 0.22 for the formation of $\mathrm{CN}^{-}$in the $v=0,1$, and 2 states, respectively. The results are compared to the recent multidimensional ab initio calculations of Chourou and Orel [Phys. Rev. A 80, 032709 (2009)].
\end{abstract}

PACS number(s): $34.80 . \mathrm{Ht}$

\section{INTRODUCTION}

We present measurements of absolute partial cross sections for formation of the $\mathrm{CN}^{-}$anion by dissociative electron attachment (DEA) to hydrogen cyanide,

$$
e^{-}(E)+\mathrm{HCN} \longrightarrow\left\{\mathrm{HCN}^{-}\right\} \longrightarrow \mathrm{H}+\mathrm{CN}^{-}(v),
$$

and to its deuterated analog DCN. The study is motivated by the current interest of theory in this process [1] and by its possible role as an initiator of abiotic synthesis of complex organic molecules in interstellar media and atmospheres of extraterrestrial bodies $[2,3]$. DEA to $\mathrm{HCN}$ was also proposed to play an important role in Titan's atmosphere [4].

Electron collisions with $\mathrm{HCN}$ were studied several times. An early DEA study is that of Inoue [5], who measured it both at low and at high pressures and reported the formation of $\mathrm{CN}^{-}$as the only primary dissociation channel, with one strong band with onset at $1.8 \pm 0.25 \mathrm{eV}$ and peak at $2.5 \mathrm{eV}$. Comparison with the $\mathrm{O}^{-} / \mathrm{CO}$ signal permitted an estimate of the $\mathrm{CN}^{-} / \mathrm{HCN}$ cross section to be $2 \times 10^{-16} \mathrm{~cm}^{2}$.

Absolute elastic cross sections were measured by Srivastava et al. [6] at energies of 3.5, 11.6, 21.6, and $50 \mathrm{eV}$ and angles of from $20^{\circ}$ to $130^{\circ}$. Burrow et al. [7] reported the electron transmission spectrum and found a broad and structureless $\pi^{*}$-shape resonance centered at $2.26 \mathrm{eV}$. The spectrum did not exhibit any vibrational (boomerang) motion of the temporary anion $\mathrm{HCN}^{-}$. Edard et al. [8] reported relative vibrational excitation cross sections, both as a function of scattering angle from $20^{\circ}$ to $125^{\circ}$, and of electron energy in the range $0.8-$ $9 \mathrm{eV}$. Bands were observed at 2.3 and $6.7 \mathrm{eV}$, respectively, and assigned to $\pi^{*}$ and $\sigma^{*}$-shape resonances.

Electron collisions with HCN were studied theoretically by Jain and Norcross [9,10] and Varambhia and Tennyson [11]. The calculation by Jain and Norcross located the $\pi^{*}$-shape resonance at $2.7 \mathrm{eV}$, about $0.4 \mathrm{eV}$ higher than the measured value of Burrow et al. [7], with an autodetachment width of $1.9 \mathrm{eV}$ at the equilibrium geometry of $\mathrm{HCN}$, in accord with the experimental results of Burrow et al. [7] and of Edard et al. [8], indicating no evidence for vibrational motion of the temporary anion. They also report a broad ${ }^{2} \Sigma$ resonance, but only when the $\mathrm{CH}$ or $\mathrm{CN}$ bonds are stretched beyond their equilibrium position. Varambhia and Tennyson [11] applied the $R$-matrix method to study electron scattering from HCN. They confirm the ${ }^{2} \Pi$-shape resonance, ranging from 2.46 to $3.27 \mathrm{eV}$ with a width between 1.14 and $1.64 \mathrm{eV}$ depending on the model used.

Chourou and Orel calculated the resonance parameters (energy and width) using the complex Kohn method in a threedimensional space and then propagated a nuclear wave packet on the resulting complex potential hypersurface and derived quantitative DEA cross sections from the intensity of the outgoing flux $[1,12]$. The results revealed many similarities, but also unexpected differences between $\mathrm{HCN}$ and the closely related case of $\mathrm{HCCH}$, which they calculated earlier [13,14] and which was also validated against absolute measurements from our laboratory $[15,16]$. The predictions are that the cross section should be substantially larger $(370 \times)$ for $\mathrm{HCN}$ than for $\mathrm{HCCH}$, and that, although the resonant potential surface of $\mathrm{HCN}$ is very similar to that of $\mathrm{HCCH}$ in the sense that the anion is bent, the bending is predicted to be less important for the dynamics in $\mathrm{HCN}$, the major path being tunneling through the barrier at nearly linear geometry. The present work aims at testing the theoretical predictions for HCN.

Bradforth et al. [17] measured the electron affinity of the $\mathrm{CN}$ radical to be $3.862 \pm 0.004 \mathrm{eV}$ and the vibrational frequency of $\mathrm{CN}^{-}$to be $2035 \pm 40 \mathrm{~cm}^{-1}$. The $\mathrm{H}^{+}+\mathrm{CN}^{-}$ion-pair formation threshold was measured with very high precision to be $122244 \pm 4 \mathrm{~cm}^{-1}(15.156 \mathrm{eV})$ by $\mathrm{Hu}$ et al. [18]. The threshold energy for the formation of $\mathrm{H}$ and $\mathrm{CN}^{-}$from $\mathrm{HCN}$ can be calculated from this number as $E_{\mathrm{th}}=1.550 \mathrm{eV}$.

\section{EXPERIMENT}

The absolute cross sections were measured with a recently constructed instrument [16], operated in the time-of-flight (TOF) mode. In this instrument a short (200 ns) pulse of electrons is sent through a target chamber with a quasistationary sample gas at a temperature of $333 \mathrm{~K}$. A 4- $\mu$ s-long pulse with an amplitude of $-300 \mathrm{~V}$, applied to a repeller about $200 \mathrm{~ns}$ later, sends the anions into a TOF tube through a slit in the wall of the target chamber. The experiment is repeated at a rate of $20 \mathrm{kHz}$. The TOF tube consists of a three-cylinder electrostatic lens, which images the ion exit slit onto the MCP detector (anion impact energy $3 \mathrm{keV}$ ). The pressure in the collision chamber is measured by a capacitance manometer and was kept typically in the range $(1-6) \times 10^{-4}$ mbar. The electron beam current 
was typically 20-80 nA (measured without pulsing) and the resolution $200 \mathrm{meV}$. The absolute calibration was against the 4.4-eV band of $\mathrm{O}^{-}$production from $\mathrm{CO}_{2}$, for which the cross section of $14.0 \mathrm{pm}^{2}$ was used as in our previous work. The setup was repeatedly verified by measuring the DEA cross sections for $\mathrm{O}^{-}$formation from $\mathrm{N}_{2} \mathrm{O}$. The results, 21 individual measurements for $\mathrm{HCN}$ and 18 measurements for $\mathrm{DCN}$, agreed within a standard deviation of $9.8 \%$ for $\mathrm{HCN}$ and $11 \%$ for $\mathrm{DCN}$. The error of the present relative measurement is thus taken as $\pm 20 \%$ for $\mathrm{HCN}$ and $\pm 25 \%$ for $\mathrm{DCN}$ and has to be combined with the error of the absolute $\mathrm{O}^{-} / \mathrm{CO}_{2}$ cross section used for normalization. The latter is taken as $\pm 15 \%$ and this results in an error bar (two standard deviations) estimate of $\pm 25 \%$ for $\mathrm{HCN}$ and $\pm 30 \%$ for DCN.

Higher resolution $(60-\mathrm{meV})$ spectra were obtained by recording relative spectra with a trochoidal electron monochromator and a quadrupole mass spectrometer (QMS) $[19,20]$ and normalizing them to the absolute values measured by the TOF experiment. In this instrument ions created by DEA in a target chamber, at a temperature of $358 \mathrm{~K}$, are drawn out through a hole in the target chamber by a three-element lens and focused onto a QMS. The base pressure of the apparatus was about $5 \times 10^{-7}$ mbar, sample gas pressure in the target chamber was around $1 \times 10^{-3}$ mbar.

The energy scales of both the TOF and the QMS experiments were calibrated on the $\mathrm{O}^{-} / \mathrm{CO}_{2}$ DEA band such that the point where the $\mathrm{O}^{-}$signal reached $50 \%$ of the height of the first peak (leading to $\mathrm{CO}$ in the $v=0$ state [20-22]) was set to have the threshold energy of $3.99 \mathrm{eV}$.

Vibrational cross section was recorded with a hemi spherical-analyzer electron spectrometer [23]. Interestingly, HCN substantially enhanced electron emission of the filaments, both thoria- and yttria-coated iridium ribbons, by a factor of 2 or more, in all three instruments. Surprisingly, DCN did not have this beneficial effect.

$\mathrm{HCN}$ and DCN have been prepared following the technique described by Keedy [24] by adding $\mathrm{H}_{2} \mathrm{SO}_{4}$ and $\mathrm{D}_{2} \mathrm{SO}_{4}$, respectively, from a dropping funnel to $\mathrm{KCN}$ in a two-neck round-bottom flask, under a stream of helium as a carrier gas. $\left(\mathrm{N}_{2}\right.$ and Ar are not suitable as carrier gases because they may condense in the trap at liquid nitrogen temperature and lead to dangerous overpressure when the trap is warmed up to $-80^{\circ} \mathrm{C}$ with closed valves.) The apparatus was flushed with helium for about $2 \mathrm{~h}$ prior to the reaction to remove water. The mixture was heated after all acid was added. The products were trapped in a two-valve trap cooled by liquid nitrogen and then transferred under vacuum to a test tube equipped with a PTFE vacuum valve. Small amount of volatile side-products were pumped-off at $-80^{\circ} \mathrm{C}$. They were identified by residual gas analyzers and by electron energy loss spectroscopy to be $\mathrm{CO}_{2}, \mathrm{C}_{2} \mathrm{~N}_{2}, \mathrm{SO}$, and $\mathrm{SO}_{2}$. The sample tube was kept in ice during the measurements and gas flow was regulated by a needle valve. All three instruments used in the present study are equipped with QMS residual gas analyzers and sample purity, in particular isotopic purity, were monitored continuously. The products were also verified by HeI photoelectron spectroscopy. It was necessary to introduce a weak stream of DCN into the instruments for up to 2 days to passivate the inlet system, after which DCN isotopic purity of $93 \%$ was reached.
TABLE I. Peak cross sections for the formation of $\mathrm{CN}^{-}$from $\mathrm{HCN}$ and DCN and their ratios (the isotope effect).

\begin{tabular}{lccc}
\hline \hline & Energy & TOF tube & Theory [1] \\
\hline $\mathrm{HCN}$ & $1.86 \mathrm{eV}$ & $940 \mathrm{pm}^{2} \pm 25 \%$ & $2800 \mathrm{pm}^{2}$ \\
DCN & $1.88 \mathrm{eV}$ & $340 \mathrm{pm}^{2} \pm 30 \%$ & $215 \mathrm{pm}^{2}$ \\
Ratio & & 2.76 & 13 \\
\hline \hline
\end{tabular}

\section{RESULTS AND DISCUSSION}

The peak cross sections for the $\mathrm{CN}^{-}$formation from $\mathrm{HCN}$ and DCN as measured with the TOF instrument are listed and compared to the theoretical values in Table I. Theory is correct in predicting that the cross section for $\mathrm{HCN}$ is much larger (measured $270 \times$, calculated $370 \times$ ) than that in the related case of acetylene $[12,13,15,16]$. The fact that the calculated cross section is $3 \times$ larger than the measured one can be considered as encouraging in view of the very steep dependence of the cross section on the calculated parameters of the resonance. That is, it appears that the theory is fundamentally correct, but that very high precision of the calculated potential hypersurface is required. On the other hand, the disagreement in the isotope effect is unexpected in view of the success of the same theory in reproducing the isotope effect in the case of acetylene [14].

Figure 1 shows the cross sections as a function of energy, measured under higher resolution with the QMS instrument and normalized to the absolute values determined with the TOF spectrometer. The QMS instrument enhances the detection of ions formed with very low kinetic energies, and the spectra from this instrument may be distorted when significant kinetic energy release occurs in the DEA. In the present case, however, any kinetic energy released will be given nearly exclusively to the $\mathrm{H}$ fragment, and no distortion occurs. This is confirmed by the fact that the (lower resolution) spectra from the TOF instrument (not shown) agree in terms of width and peak position with those from the QMS instrument.

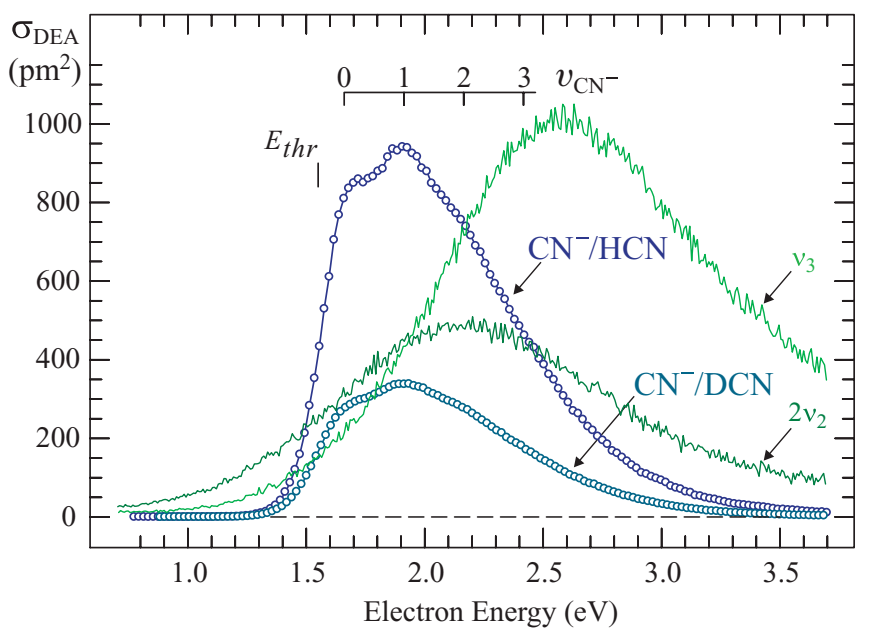

FIG. 1. (Color online) Cross sections for the yields of $\mathrm{CN}^{-}$from $\mathrm{HCN}$ and DCN. The grid above the DEA spectra indicates vibrational spacings of the $\mathrm{CN}^{-}$ion. The intrinsic position and shape of the ${ }^{2} \Pi$ resonance is indicated by the cross sections for the excitation of the $v_{3}\left(\mathrm{CN}\right.$ stretch) and $2 v_{2}$ (bend) vibrations of $\mathrm{HCN}$. 
The low-energy side of the band is vertical or nearly vertical. We determine the width of the rise as the difference between the energies where the signal reaches $25 \%$ and $75 \%$ of its peak intensity, respectively. The width is measured to be about $110 \mathrm{meV}$ for HCN and DCN, this is only slightly larger than the value of $80 \mathrm{meV}$ measured for the $\mathrm{O}^{-}$band from $\mathrm{CO}_{2}$. The onset $(50 \%$ height $)$ is at the energetical threshold $(1.550 \mathrm{eV})$ for the process, there is no activation energy.

Both the onset and the peak of the band are at somewhat lower energies than those reported by Inoue [5], but in view of the absence of electron monochromator and the relatively crude method of energy scale calibration of that early work, the differences are not alarming and the present values are to be preferred. The band can be assigned to the ${ }^{2} \Pi$ resonance observed in the transmission experiment of Burrow et al. [7] and the vibrational excitation experiment of Edard et al. [8]. A comparison of the DEA and the vibrational excitation bands (the latter are indicative of the inherent shape of the ${ }^{2} \Pi$ resonance) in Fig. 1 indicates that the DEA occurs at the lowenergy end of the resonance. The DEA band is narrower and peaks at lower energy than the VE bands. This is a commonly encountered phenomenon whereby an electron attachment at higher energies leads to less DEA signal because $\Gamma$ is larger there and the nuclei have to travel a longer distance to reach the stabilization point, leading to a less favorable competition of dissociation with autodetachment. Interestingly, the cross section for the excitation of the bending vibrations peaks at lower energies $(2.16 \mathrm{eV})$ than that of the $\mathrm{CN}$ stretch vibration $(2.57 \mathrm{eV})$.

Interesting is the observation of broad structure on both the $\mathrm{HCN}$ and the DCN DEA spectra which we interpret as due to opening of dissociation channels leading to vibrationally excited $\mathrm{CN}^{-}$. These structures cannot be due to vibrational (boomerang) structure of the $\mathrm{HCN}^{-}$resonance, because both the vibrational excitation cross section and the transmission spectrum of Burrow lack any vibrational structure. The spacing of the structures (although it cannot be determined precisely because of their large widths) agrees with the $\mathrm{CN}^{-}$vibrational spacing, indicated by a grid in Fig. 1. A related case of structures at thresholds for vibrationally excited products was reported in the $\mathrm{O}^{-} / \mathrm{CO}_{2}$ DEA [20-22], with two important differences. In the $\mathrm{CO}_{2}$ case it is the neutral $\mathrm{CO}$ fragment which is vibrationally excited, whereas it is the $\mathrm{CN}^{-}$anion in the case $\mathrm{HCN}$. The vibrational levels of $\mathrm{CN}^{-}$in question $(v=0-3)$ are not autodetaching because of the large electron affinity of $\mathrm{CN}(3.862 \mathrm{eV})$. The second difference is that in the case of $\mathrm{HCN}$ the excess energy is given almost entirely to the neutral $\mathrm{H}$ fragment so that it is not possible to enhance the threshold structures by detecting only ions with (nearly) zero kinetic energy. This is in contrast to the $\mathrm{CO}_{2}$ case where the threshold structure could be made to appear much more clearly in this way [20]. Vibrationally excited fragment anions have been detected also in the case of the $\mathrm{O}_{2}{ }^{-} / \mathrm{O}_{3}$ [25] except that the much lower electron affinity of $\mathrm{O}_{2}(0.44 \mathrm{eV})$ made the vibrationally excited $\mathrm{O}_{2}{ }^{-}$autodetach-it was detected by the emitted electrons.

The structures are interesting because they permit determination of the branching ratios into the different final vibrational states and thus open an additional dimension for potential comparison with theory. To obtain the cross sections

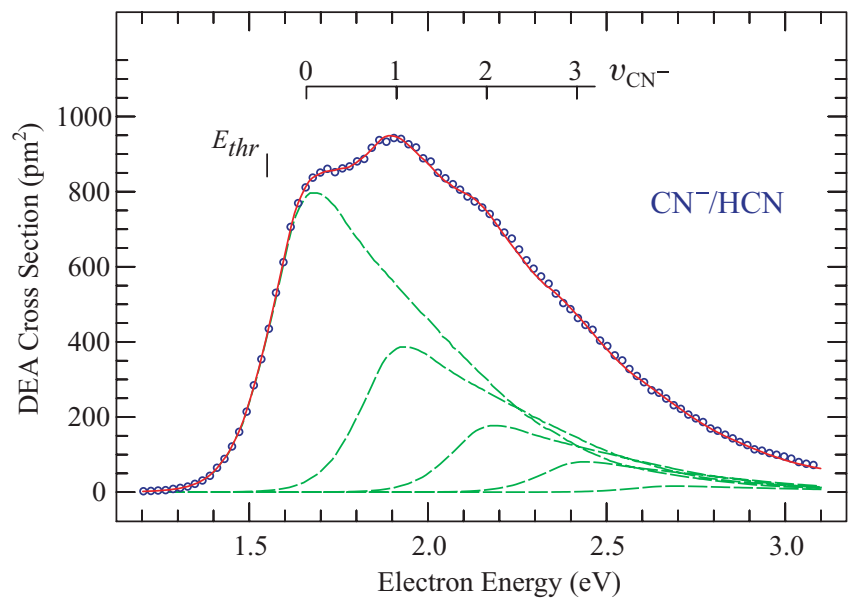

FIG. 2. (Color online) Decomposition of the $\mathrm{CN}^{-} / \mathrm{HCN}$ cross section into parts corresponding to formation of $\mathrm{CN}^{-}$in individual vibrational levels $v\left(\mathrm{CN}^{-}\right)$. Experimental data are shown as circles, the individual contributions as dashed (green) lines, and their sum as a continuous (red) line. The relative heights for the $v=0,1$, and 2 profiles are $1.0,0.49$, and 0.22 .

for $\mathrm{CN}^{-}$in the individual vibrational states, a profile of the individual contribution was found empirically such that a sum of these profiles, shifted by the appropriate quanta of the $\mathrm{CN}^{-}$ vibrational frequency and multiplied by suitable factors, fitted the experimental data, as shown in Fig. 2. The relative factors for $v=0,1$, and 2 are 1, 0.49, and 0.22 for $\mathrm{HCN}$ and 1, 0.515, and 0.25 for DCN, respectively. Within confidence limits the factors are the same for $\mathrm{HCN}$ and DCN.

\section{CONCLUSIONS}

An important current quest in the field of electron-molecule collisions and electron-driven chemistry is the development of a method to calculate $a b$ initio cross sections for dissociative electron attachment in polyatomic molecules. The present work, together with the earlier similar work on acetylene $[15,16]$, makes a contribution to this effort by providing quantitative data to assess theoretical predictions. The theoretical predictions for acetylene $[13,14]$ were confirmed by the experiment within a factor of 2 in terms of the absolute value, and, when the initial thermal vibrational excitation of the target was also taken into account, the experimental isotope effect of about 15 was reproduced correctly by the theory.

$\mathrm{HCN}$ is interesting because, despite many similarities in terms of a low-lying $\pi^{*}$-shape resonance, the fact that the negative ion in this state is bent, and that there is an energy barrier for dissociation in the linear geometry which decreases, and even disappears, in the bent geometry, the theoretical study predicted dramatic differences [1]. In terms of mechanism, the theory predicts that, whereas in the case of acetylene the negative ion bent and the wave packet traveled around the barrier, in $\mathrm{HCN}$ bending is minimal and tunneling through the barrier dominates.

The DEA cross section of $\mathrm{HCN}$ was predicted to be $370 \times$ larger than in acetylene at $300 \mathrm{~K}[1,14]$. This prediction is confirmed quite well by the present study, our measured value for $\mathrm{HCN}$ is $270 \times$ larger than our measured value in acetylene, 
at $333 \mathrm{~K}$. On the absolute scale, the measured values are lower than calculated, by a factor of 2 for acetylene, and by a factor of 3 for HCN. When comparing the cross sections it must be borne in mind, however, that the cross sections depend very steeply on the calculated width $\Gamma$ of the resonance-changing the width by $7 \%$ changed the cross section by more than $50 \%$ in Ref. [14] - and on the calculated height and width of the energy barrier through which the $\mathrm{H}$ atom has to tunnel. In view of this steep dependence of the cross section on the resonance parameters, the disagreement between the measured and calculated values is not alarming. Surprising is, however, the discrepancy of the HCN/DCN DEA cross-section ratios, measured to be 2.8 and calculated as 13 (Table I).

There are differences between the calculation and the experiment other than the cross-section magnitudes. The present VE cross section, indicative of the position of the ${ }^{2} \Pi$ resonance, peak at $2.16 \mathrm{eV}$ (bending) and $2.57 \mathrm{eV}$ (CN stretch), whereas the calculated resonance is centered around $3.5 \mathrm{eV}$ [1]. A second point is that the present cross sections have a nearly vertical onset at threshold $(1.55 \mathrm{eV})$ and peak around $1.85 \mathrm{eV}$, whereas the calculated cross sections have a very gradual onset and peak much higher, around $3 \mathrm{eV}$ [1]. This could be a consequence of the calculated resonance energy being about $1 \mathrm{eV}$ higher than measured, but it could also mean that the dependence of the width $\Gamma$ on energy may be important.

\section{ACKNOWLEDGMENTS}

This research is part of contract 200020-113599/1 of the Swiss National Science Foundation and of COST Action CM0601.
[1] S. T. Chourou and A. E. Orel, Phys. Rev. A 80, 032709 (2009).

[2] S. Gupta, E. Ochiai, and C. Ponnamperuma, Nature 293, 725 (1981).

[3] R. V. Yelle, Astrophys. J. 383, 380 (1991).

[4] V. Vuitton, P. Lavvas, R. V. Yelle, M. Galand, A. Wellbrock, G. R. Lewis, A. J. Coates, and J.-E. Wahlund, Planet. Space Sci. 57, 1558 (2009).

[5] M. Inoue, J. Chim. Phys. 63, 1061 (1966).

[6] S. K. Srivastava, H. Tanaka, and A. Chutjian, J. Chem. Phys. 69, 1493 (1978).

[7] P. D. Burrow, A. E. Howard, A. R. Johnston, and K. D. Jordan, J. Phys. Chem. 96, 7570 (1992).

[8] F. Edard, A. P. Hitchcock, and M. Tronc, J. Phys. Chem. 94, 2768 (1990).

[9] A. Jain and D. W. Norcross, Phys. Rev. A 32, 134 (1985).

[10] A. Jain and D. W. Norcross, J. Chem. Phys. 84, 739 (1986).

[11] H. N. Varambhia and J. Tennyson, J. Phys. B: At. Mol. Opt. Phys. 40, 1211 (2007).

[12] S. T. Chourou and A. E. Orel, J. Phys. Conf. Ser. 194, 052032 (2009).
[13] S. T. Chourou and A. E. Orel, Phys. Rev. A 77, 042709 (2008).

[14] S. T. Chourou and A. E. Orel, Phys. Rev. A 80, 034701 (2009).

[15] O. May, J. Fedor, B. C. Ibănescu, and M. Allan, Phys. Rev. A 77, 040701(R) (2008).

[16] O. May, J. Fedor, and M. Allan, Phys. Rev. A 80, 012706 (2009).

[17] S. E. Bradforth, E. H. Kim, D. W. Arnold, and D. M. Neumark, J. Chem. Phys. 98, 800 (1993).

[18] Q. J. Hu, Q. Zhang, and J. W. Hepburn, J. Chem. Phys. 124, 074310 (2006)

[19] M. Stepanović, Y. Pariat, and M. Allan, J. Chem. Phys. 110, 11376 (1999).

[20] R. Dressler and M. Allan, Chem. Phys. 92, 449 (1985).

[21] A. Stamatovic and G. J. Schulz, Phys. Rev. A 7, 589 (1973).

[22] P. Cicman, G. Senn, G. Denifl, D. Muigg, J. D. Skalny, P. Lukac, A. Stamatovic, and T. D. Märk, Czech. J. Phys. 48, 1135 (1998).

[23] M. Allan, J. Phys. B: At. Mol. Opt. Phys. 40, 3531 (2007).

[24] C. R. Keedy, J. Chem. Educ. 69, A296 (1992).

[25] M. Allan, K. R. Asmis, D. B. Popovic, M. Stepanovic, N. J. Mason, and J. A. Davies, J. Phys. B: At. Mol. Opt. Phys. 29, 3487 (1996). 